$\rightarrow$ NEURONAL PLASTICITY

\section{Mossy fibres WNT more contact with CA3}

Living in a stimulating environment has cognitive benefits for humans and animals alike, and hippocampal neurons in particular show structural plasticity in response to environmental manipulations. Gogolla et al. now show that environmental enrichment produces a net global increase in synapse densities in the hippocampus, which at mossy-fibre-CA3 synapses depends on WNT signalling.

The authors used mice expressing green fluorescent protein in neuronal membranes, which allowed

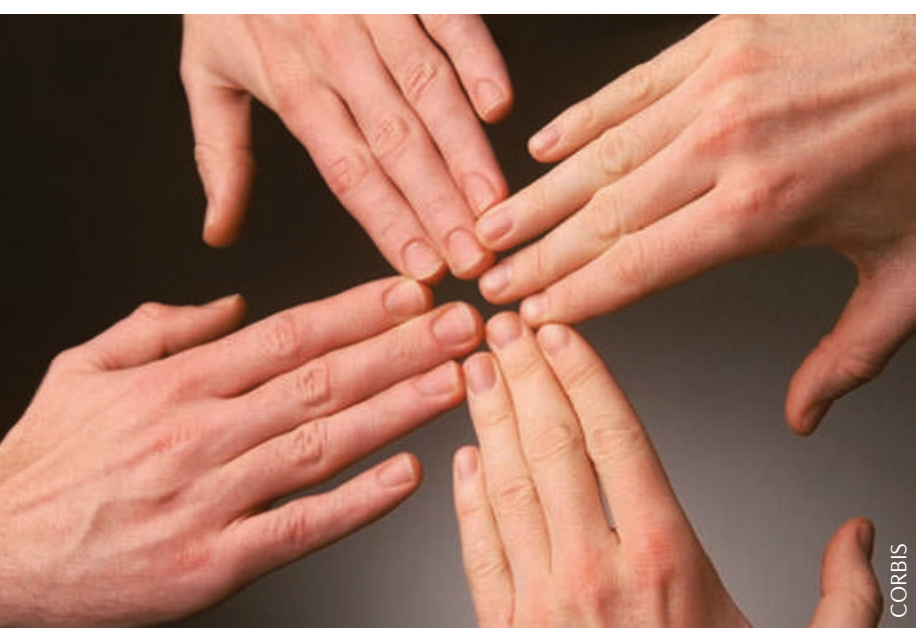

visualization of mossy fibres and their large mossy fibre terminals (LMT) onto CA3 pyramidal cells. They found that 3 weeks of environmental enrichment increased both the total number of synapses in the stratum lucidum and the density of LMT synapses onto pyramidal neurons. Because WNT proteins are known to be involved in synaptogenesis and plasticity, the authors investigated whether they contribute to enrichment-induced structural plasticity. They detected WNT7a/b immunoreactivity in hippocampal pyramidal cells and interneurons throughout the hippocampus but not in mossy fibres, which indicates that WNT is expressed postsynaptically at mossy-fibre-CA3 synapses. Importantly, environmental enrichment increased WNT7b levels specifically in CA3 pyramidal cells.

Administration of a WNT signalling inhibitor directly into CA3 throughout the enrichment procedure suppressed its effects on synapse numbers, whereas recombinant WNT7a or a WNT-signalling agonist in control mice (that is, without environmental enrichment) mimicked them. After termination of the enrichment procedure, LMT synapse numbers and CA3 WNT7a/b levels eventually returned to their baseline; inhibition of WNT signalling after environmental enrichment accelerated this reversal, suggesting that WNT7 signalling is required to both induce and maintain the enrichmentinduced synaptic increases.

Suppressing synaptic activity in hippocampal slices with the sodiumchannel blocker TTX reduced WNT7a/b immunoreactivity in CA3 neurons, whereas enhancing it with GABA receptor antagonists increased it. Together, the findings from this study indicate that environmentalenrichment-induced excitatory activity increases postsynaptic WNT7a/b levels, and the resulting WNT signalling in turn regulates LMT synapse numbers.

Improved spatial learning and memory is a well-established effect of environmental enrichment. Considering the crucial role of mossy-fibre input to CA3 pyramidal cells in pattern separation, WNT may be a key player in at least one of the cognitive effects of experiencedependent plasticity.

Leonie Welberg

ORIGINAL RESEARCH PAPER Gogolla, N. et al. Wnt signaling mediates experience-related regulation of synapse numbers and mossy fiber connectivities in the adult hippocampus. Neuron $62,510-525(2009)$ 